\title{
ANTIMICROBIAL SUSCEPTIBILITY AMONG URINARY ESCHERICHIA COLI ISOLATES FROM FEMALE OUTPATIENTS: AGE-RELATED DIFFERENCES
}

\author{
Zorana Djordjević ${ }^{1}$, Marko Folić ${ }^{2,3}$, Violeta Ninković4, Dragan Vasiljević ${ }^{3,4}$, Slobodan Janković2,3 \\ 'Department of Hospital Infections Control, Clinical Centre Kragujevac, Kragujevac, Serbia \\ ${ }^{2}$ Clinical Pharmacology Department, Clinical Centre Kragujevac, Kragujevac, Serbia \\ ${ }^{3}$ Faculty of Medical Sciences, University of Kragujevac, Kragujevac, Serbia \\ ${ }^{4}$ Public Health Institute Kragujevac, Kragujevac, Serbia
}

\section{SUMMARY}

Objectives: Urinary tract infections (UTIs) are common problems in women, and important reason for visiting primary care physicians, resulting in substantial financial burden to community. The aim of this study was to determine the resistance rates of $E$. coli to commonly prescribed antimicrobial drugs for community-acquired UTIs in women and to establish the association between age and resistance to antibiotics among isolates of $E$. coli from urine.

Methods: The study was designed as a retrospective cross-sectional study during the 5-years period. It was conducted on a sample of urinary tract isolates of $E$. coli taken from women with community-acquired UTIs. After prevalence of $E$. coli resistance to antibiotics was established, the analysis of risk factors for emergence of resistance was conducted.

Results: There were 10,734 isolates of $E$. coli, comprising $70.62 \%$ of all samples analyzed. E. coli was the most frequently resistant to ampicillin $(54.68 \%)$, followed by trimethoprim-sulphamethoxazole (37.46\%), first and second generation cephalosporins (cephalexin and cefaclor) $(29.53 \%$ both), and ciprofloxacin $(23.80 \%)$. Less than $50 \%$ of $E$. coli isolates was sensitive to all three tested antibiotics, and nearly $13 \%$ acquired tripleresistance. Prevalence of isolates resistant to two or three agents was higher in the subgroup of women older than 65 years.

Conclusions: Empirical choice of antimicrobial agent for community-acquired non-complicated UTIs in women should be individualized on the basis of the patient's age, prevalence of resistance in the local community, and compliance history of the patient.

Key words: urinary tract infections, community acquired, E. coli, resistance, utilization

Address for correspondence: M. Folic, Faculty of Medical Sciences, University of Kragujevac, 69 Svetozara Markovica Street, 34000 Kragujevac, Serbia. E-mail: markof@medf.kg.ac.rs

https://doi.org/10.21101/cejph.a4833

\section{INTRODUCTION}

Urinary tract infections (UTIs) are common problems in women, and important reason for visiting primary care physicians, resulting in substantial financial burden to community. According to announcement from the Ambulatory Medical Care in 2007, there were over 8.6 million visits to general practitioners per year in the USA, and $85 \%$ of the patients were women (1). UTI occurs four times more frequently in females than in males due to anatomical differences and the highest incidence is between 16 and 35 years of age. Around 10\% of women suffer from an infection yearly and more than 40 to $60 \%$ acquire infection at some point in their lives (2). The UTI is actually whole spectrum of clinical entities, but the most common is acute cystitis. Community-acquired uncomplicated cystitis in women is monomicrobial and in more than $80 \%$ of cases caused by Enterobacteriaceae, especially by E. coli which is isolated from approximately $70-95 \%$ of urine samples $(3,4)$.
Modern treatment of uncomplicated cystitis in women in most countries is empirical, administering antibiotics or uroantiseptics to patients without results from urine culture, aiming to achieve fast resolution of symptoms $(5,6)$. However, it is necessary to have good knowledge of spectrum of possible aetiological agents and their susceptibility patterns. Recent reports received from different regions of the world indicate an increase in the frequency of UTI pathogens resistant to commonly used antibiotics, which is the most likely due to their inappropriate use. The occurrence of resistant and multidrug-resistant strains is major health problem all over the world because it limits the choice of antibiotics and increases the treatment costs. As already mentioned, good knowledge of local and national antimicrobial resistance patterns of the $E$. coli is of the utmost importance in order to implement evidence-based recommendations in empiric antibiotic treatment of UTIs in women.

The main objectives of this study were to determine the resistance rates of $E$. coli to commonly prescribed antimicrobial 
drugs for community-acquired UTIs in women and to establish the association between age and resistance to antibiotics among isolates of $E$. coli from urine. In addition, the study aim was to examine the impact of antibiotic utilization to resistance rates of tested isolates.

\section{MATERIALS AND METHODS}

\section{Study Design}

We conducted a retrospective cross-sectional study during the period from January 2009 to December 2013 (5 years).

\section{Data Collection}

The data presented were obtained from the Department of Microbiology, Institute of Public Health in Kragujevac, Serbia, which analyzed urine samples taken from women with symptomatic UTIs. This department has internal quality control procedures and participates in the External Programme for Quality Assurance by the United Kingdom National External Quality Assessment Service for Microbiology (UK NEQAS) and by the Institute of Public Health of Belgrade, Serbia. The urine samples were taken from the outpatients coming from an area with about 200,000 inhabitants. The following data were taken from each patient: date when the sample was taken, age, gender, urine culture results, identification of the bacterial strain responsible for the UTI, and results of the corresponding Antimicrobial Susceptibility Test (AST). Clinical data related to the isolates were not available.

As part of routine procedure, the patients were given detailed instructions about proper sampling of urine to avoid contamination by microorganisms, including clean-catch technique after usual daily hygiene of genital area. The urine sample was collected from middle portion of the micturition stream directly into a sterile recipient early in the course of the disease. Transport to the laboratory and analyses of samples were performed within the two hours after collection of a sample. When this was not possible the urine samples were stored at $4{ }^{\circ} \mathrm{C}$ and processed within the next 24 hours. Identification of the microorganisms was made by plating on chromogenic CPS agar (Biomerieux, France) and by incubation for $18-24$ hours at $35 \pm 2{ }^{\circ} \mathrm{C}$, as well as by performing relevant biochemical tests appropriate for bacterial confirmation.

For this study the database was searched for data on E. coli isolates from women with at least one episode of communityacquired UTI. The gold standard for diagnosis of UTI was positive culture with more than $\geq 10^{5} \mathrm{CFU} / \mathrm{mL}$ of $E$. coli. The patients whose urine samples were obtained by catheterization and those with repeated testing of $E$. coli susceptibility were excluded from the study.

All isolates E. coli were tested for susceptibility to antimicrobial drugs. The AST was made by disk-diffusion method on Mueller-Hinton Agar (Biomerieux, France) and interpreted according to the guidelines of the Clinical and Laboratory Standards Institute (CLSI), formerly the National Committee for Clinical Laboratory Standards (NCCLS) (7). Sensitivity was determined by measuring the diameter of the zones of inhibition and isolates were classified as either susceptible or not susceptible (intermediate or resistant) for the following tested antibiotics: ampicillin (25 $\mu \mathrm{g} / \mathrm{mL})$, cephalexin $(30 \mu \mathrm{g} / \mathrm{mL})$, cefaclor $(30 \mu \mathrm{g} / \mathrm{mL})$, cefotaxime
$(30 \mu \mathrm{g} / \mathrm{mL})$, ceftriaxone $(30 \mu \mathrm{g} / \mathrm{mL})$, trimethoprim-sulphamethoxazole $(2.5 \mu \mathrm{g} / \mathrm{mL})$, gentamicin $(10 \mu \mathrm{g} / \mathrm{mL})$, amikacin $(30 \mu \mathrm{g} /$ $\mathrm{mL})$, ciprofloxacin $(5 \mu \mathrm{g} / \mathrm{mL})$, and ofloxacin $(5 \mu \mathrm{g} / \mathrm{mL})$.

\section{Utilization of Antibiotics}

Data on utilization of antibiotics (cephalexin, ciprofloxacin and trimethoprim-sulphamethoxazole) were taken from annual reports on "Trade and Utilization of Drugs" made by the Agency for Medicines and Medical Devices of Serbia (8). According to the World Health Organization (WHO) recommendations, the volume of utilization of drugs is converted into the number of Defined Daily Doses (DDD) per 1,000 inhabitants per day. DDD is a statistical unit of a medicinal product, whose value represents the average daily dose for the main indication of the drug administration in adults. It allows the comparison of the utilization of different drugs whatever their differences in the mean prescribed daily dose were.

\section{Statistical Methods}

Primary analysis of collected data was made by descriptive statistics. Values of continuous variables were described by measures of central tendency (mean or median) and that of dispersion (standard deviation from the mean and range). Values of categorical variables were described by rates and percentages. Difference in values of a categorical variable among the study groups was tested for significance by Chi-square test (e.g. differences in resistance rates for age groups and antibiotype of resistance). For additional assessment of influence of the study variables on resistance patterns in samples from women, the univariate logistic regressions was used. Besides, correlation between the resistance rates to certain antibiotics and their utilization figures was tested for significance. Statistical hypotheses were considered true if probability of null-hypothesis was less than 0.05 . All calculations were performed by the statistical software SPSS (SPSS Inc, ver.18, Chicago, IL).

\section{RESULTS}

During the study period there were 10,734 isolates of E. coli, comprising $70.62 \%$ of all samples analyzed in the study population. The results of susceptibility according to the basic CLSI interpretative categories are shown in Table 1. E. coli was the most frequently resistant to ampicillin $(54.68 \%)$, followed by trimethoprim-sulphamethoxazole (TMP-SXT) (37.46\%), first and second generation cephalosporins (cephalexin and cefaclor) (29.53\% both) and ciprofloxacin $(23.80 \%)$. Amikacin and third generation cephalosporins had the lowest resistance rates for the E. coli strains.

Out of the total number of $E$. coli isolates, $21.75 \%$ belonged to the group of women 18-45 years old, one third to the group of women 46-65 years old, and in the oldest age group there was nearly half of all tested isolates (Table 2). The age was identified as a significant risk factor for the development of resistance to commonly used antibiotics. In the subgroup of women older than 65 , resistance to cephalexin was $32.71 \%$, in comparison to $29.55 \%$ in the subgroup of women $46-65$ years old and to $22.73 \%$ in the subgroup of women younger than $45(\mathrm{OR}=1.263 ; 95 \%$ $\mathrm{CI}=1.193-1.337 ; \mathrm{p}<0.001)$. For TMP-SXT the resistance rates 
Table 1. Antimicrobial resistance to selected antibiotics of E. coli isolates from urine samples in women, 2009-2013 (N=10,734)

\begin{tabular}{|l|c|c|c|}
\hline Antibiotic & $\begin{array}{c}\text { Number of isolates with available } \\
\text { results }\end{array}$ & Number of resistant isolates & $\%$ \\
\hline Ampicillin & 9,978 & 5,456 & 54.68 \\
\hline Cephalexin & 9,696 & 2,863 & 29.53 \\
\hline Cefaclor & 9,649 & 2,849 & 7.53 \\
\hline Cefotaxime & 10,137 & 731 & 7.21 \\
\hline Ceftriaxone & 10,228 & 735 & 37.46 \\
\hline Trimethoprim-sulphamethoxazole & 9,950 & 3,727 & 20.14 \\
\hline Gentamicin & 9,947 & 2,003 & 5.41 \\
\hline Amikacin & 10,126 & 548 & 23.80 \\
\hline Ciprofloxacin & 9,881 & 2,352 & 21.37 \\
\hline Ofloxacin & 6,173 & 1,319 & \\
\hline
\end{tabular}

Table 2. Antimicrobial resistance rates (\%) of E. coli from urine samples in women, by age group $(N=10,734)$

\begin{tabular}{|c|c|c|c|c|}
\hline Age & $\mathrm{n}(\%)$ & $\begin{array}{c}\text { Cephalexin resistance } \\
\mathrm{n}(\%)\end{array}$ & $\begin{array}{c}\text { Trimethoprim-sulphameth- } \\
\text { oxazole resistance } \\
n(\%)\end{array}$ & $\begin{array}{c}\text { Ciprofloxacin resistance } \\
\mathrm{n}(\%)\end{array}$ \\
\hline $18-45$ years & $2,335(21.75)$ & $475 / 2,090(22.73)$ & $595 / 2,161(27.53)$ & $225 / 2,133(10.55)$ \\
\hline $46-65$ years & $3,528(32.87)$ & $936 / 3,167(29.55)$ & $1,227 / 3,235(37.93)$ & $729 / 3,242(22.49)$ \\
\hline$>65$ years & $4,871(45.38)$ & $1,452 / 4,439(32.71)$ & $1,905 / 4,554(41.83)$ & $1,398 / 4,506(31.03)$ \\
\hline Total & $10,734(100.0)$ & $2,863 / 9,696(29.53)$ & $3,727 / 9,950(37.46)$ & $2,352 / 9,881(23.80)$ \\
\hline $\begin{array}{l}\text { OR } \\
\text { (95\% Cl) } \\
\text { p-value }\end{array}$ & & $\begin{array}{c}1.263 \\
(1.193-1.337) \\
<0.001\end{array}$ & $\begin{array}{c}1.341 \\
(1.272-1.414) \\
<0.001\end{array}$ & $\begin{array}{c}1.832 \\
(1.715-1.956) \\
<0.001\end{array}$ \\
\hline
\end{tabular}

Table 3. Resistance profile of E. coli isolates from urine in women to three key antibiotics (cephalexin, trimethoprim-sulphamethoxazole and ciprofloxacin)

\begin{tabular}{|c|c|c|c|c|c|c|c|c|}
\hline \multirow[b]{2}{*}{ Antibiotype } & \multirow[b]{2}{*}{ Cephalexin } & \multirow{2}{*}{$\begin{array}{c}\text { Trimethoprim- } \\
\text { sulphamethoxazole }\end{array}$} & \multirow[b]{2}{*}{ Ciprofloxacin } & \multicolumn{3}{|c|}{ Age } & \multirow[b]{2}{*}{$p$-value } & \multirow{2}{*}{$\begin{array}{l}\text { Total } \\
\mathrm{n}(\%)\end{array}$} \\
\hline & & & & $\begin{array}{c}18-45 \text { years } \\
n(\%)\end{array}$ & $\begin{array}{c}46-65 \text { years } \\
\mathrm{n}(\%)\end{array}$ & $\begin{array}{c}>65 \text { years } \\
n(\%)\end{array}$ & & \\
\hline A & $S$ & $S$ & $S$ & $1,181(59.80)$ & $1,440(47.97)$ & $1,838(43.69)$ & $<0.001$ & $4,459(48.55)$ \\
\hline B & S & $S$ & $\mathrm{R}$ & $33(1.67)$ & $82(2.73)$ & $173(4.11)$ & $<0.001$ & $288(3.14)$ \\
\hline C & $S$ & $\mathrm{R}$ & $S$ & $276(13.97)$ & $429(14.29)$ & $510(12.12)$ & 0.004 & $1,215(13.23)$ \\
\hline D & $\mathrm{R}$ & $S$ & $S$ & $199(10.08)$ & $269(8.96)$ & $300(7.13)$ & ns & $768(8.36)$ \\
\hline$E$ & $S$ & $\mathrm{R}$ & $R$ & $46(2.33)$ & $170(5.66)$ & $320(7.61)$ & $<0.001$ & $536(5.84)$ \\
\hline$F$ & $\mathrm{R}$ & $\mathrm{R}$ & $S$ & $111(5.62)$ & $177(5.90)$ & $242(5.75)$ & ns & $530(5.77)$ \\
\hline G & $\mathrm{R}$ & $S$ & $\mathrm{R}$ & $29(1.47)$ & $60(2.00)$ & $125(2.97)$ & ns & $214(2.33)$ \\
\hline $\mathrm{H}$ & $\mathrm{R}$ & $\mathrm{R}$ & $\mathrm{R}$ & $100(5.06)$ & $375(12.49)$ & $699(16.62)$ & $<0.001$ & $1,174(12.78)$ \\
\hline Total & & & & $1,975(100.0)$ & $3,002(100.0)$ & $4,207(100.0)$ & & $9,184(100.0)$ \\
\hline
\end{tabular}

S - susceptible; R - resistant

were $41.83 \%, 37.93 \%$ and $27.53 \%$, respectively $(\mathrm{OR}=1.341$; $95 \% \mathrm{CI}=1.272-1.414 ; \mathrm{p}<0.001$ ), and for ciprofloxacin $31.03 \%$, $22.49 \%$ and $10.55 \%$, respectively $(\mathrm{OR}=1.832 ; 95 \% \mathrm{CI}=1.715-$ 1.956; $\mathrm{p}<0.001$ ) (Table 2).

Susceptibility testing to three key antibiotics (cephalexin, TMP-SXT and ciprofloxacin) was done simultaneously for 9184 $(85.56 \%)$ isolates. Nearly half of the isolates $(n=4,459 ; 48.55 \%)$ were sensitive to all three antibiotics (Table 3). Resistance to only one of the tested antibiotics was reported in 2,271 (24.73\%) isolates: $8.36 \%$ to cephalexin, $13.23 \%$ to TMP-SXT and $3.14 \%$ to ciprofloxacin. Dual resistance was seen in 1,280 (13.94\%) isolates: TMP-SXT and ciprofloxacin (5.84\%), cephalexin and TMP-SXT (5.77\%), and cephalexin and ciprofloxacin $(2.33 \%)$. Triple-resistance was observed in 1,174 (12.78\%) isolates.

Lower rates of resistance with increasing age were shown for monoresistance to ciprofloxacin (antibiotype B) with $1.67 \%$ of isolates from patients aged 18-45 years being resistant, in comparison to $2.75 \%$ for patients $46-65$ years old and $4.11 \%$ 
for females patients older than 65 years $(\mathrm{p}<0.001)$. For elderly females subgroup, statistically significant differences between age groups were also prevalent in rates of monoresistance to TMP-SXT (antibiotype C), in dual resistance rates to TMP-SXT and ciprofloxacin (antibiotype E), as well as in rates of tripleresistance to cephalexin, TMP-SXT and ciprofloxacin (antibiotype H) $(\mathrm{p}=0.004, \mathrm{p}<0.001, \mathrm{p}<0.001$, respectively). Analysis of coresistance is summarized in Table 3.

The annual variations in resistance rate (shown as \%) of $E$. coli isolates to the three most commonly used antibiotics for UTIs in the study period were also identified. Proportion of isolates not susceptible to cephalexin, ciprofloxacin and TMP-SXT was higher in the last years of observation (Figure 1, 2, 3). The increase in resistance was not correlated with the total use of antibiotics (shown as DDDs per 1,000 inhabitants, per day) (for cephalexin $r=0.100 ; p=0.873$, TMP-SXT $r=0.500 ; p=0.391$, and ciprofloxacin $r=0.600 ; p=0.285$ ).

\section{DISCUSSION}

Our study gives detailed information on patterns of antimicrobial resistance of $E$. coli, the main aetiological agents of community-acquired UTIs in women, and on commonly used drugs in

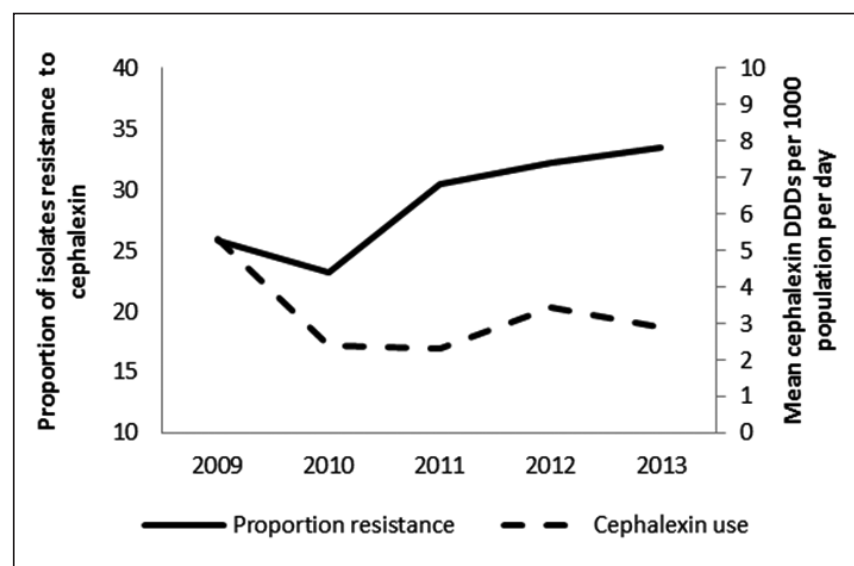

Fig. 1. Antimicrobial utilization and E. coli resistance to cephalexin.



Fig. 2. Antimicrobial utilization and E. coli resistance to trimethoprim-sulphamethoxazole (TMP-SXT).

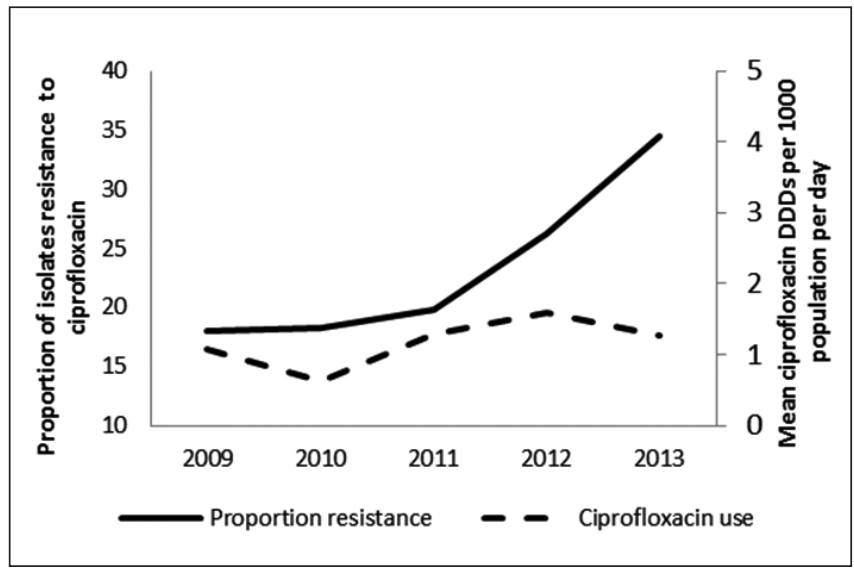

Fig. 3. Antimicrobial utilization and E. coli resistance to ciprofloxacin.

relation to age. The factors that give reliability to this study include a very large sample of isolates obtained from outpatients, which gives us an opportunity to estimate true resistance rates in community, without contaminating data from hospital patients with high resistance rates. Such studies with large number of isolates should be conducted periodically in order to estimate efficacy of empirical antibiotic therapy, especially in countries which lack national guidelines of good clinical practice for treatment of UTIs, so the physicians have to rely on various international guidelines. The principal treatment intervention in patients with UTI is oral therapy with an antibiotic effective against gramnegative aerobic coliform bacteria, such as E. coli. The newest international guideline for the treatment of uncomplicated UTI in women, sponsored by the Infectious Diseases Society of America (IDSA) (9), recommends metronidazole as the first-line therapy, then TMP-SXT (only if the local resistance E. coli is less than $20 \%$ ), nitrofurantoin and fosfomycin, but fluoroquinolones in the second-line only, because these agents have a propensity for rapid development of resistance. According to these guidelines, betalactam agents (e.g., amoxicillin-clavulanate, cefdinir, cefaclor, cefpodoxime-proxetil) are recommended as alternative therapy. Similar suggestions could be found in European Association of Urology (EAU) guidelines (10).

We showed that $37.46 \%$ E. coli isolates were resistant to TMP-SXT, while the numbers for fluoroquinolones were lower (ofloxacin $21.37 \%$ and ciprofloxacin $23.8 \%$ ), but still relatively high compared to some European countries $(11,12)$. This can be partly explained by the fact that these antibiotics have been widely used for treatment of community-acquired UTI over the past decade in this part of the world. Recently received reports from the European Union suggest that there is a growing trend of resistance rate to these antibiotics among community isolates of $E$. coli from women with acute uncomplicated UTI (13, 14). One earlier large international survey on the antimicrobial resistance of pathogens implicated in uncomplicated UTI (Antimicrobial Resistance Epidemiological Survey on Cystitis) conducted in nine European countries and Brazil in the period from 2003-2006, showed high resistance of E. coli to sulphonamide (29.4\%) and to fluoroquinolone (ciprofloxacin 8.1\%) (15). Data from geographically isolated areas (16), but also from countries such as Canada (17), where control of antibiotic prescribing was achieved, indicate that sensitivity of $E$. coli could be preserved. 
Recent studies showed an increase in uncomplicated UTI caused by extended-spectrum beta-lactamase-producing (beta-lactamresistant) strains of $E$. coli worldwide, which are also resistant to the fluoroquinolones and TMP-SXT $(18,19)$. In order to preserve their effectiveness for the treatment of serious infections German national guidelines no longer recommend these agents as first-line empiric treatment for uncomplicated cystitis (20). This speaks in favour of the restriction of their use in empirical therapy and suggests that there is need for additional efforts in order to preserve the sensitivity of the pathogens causing UTI.

High rate of resistance to ampicillin observed in our study (nearly 55\%) is in agreement with previously published data (21). Besides the high resistance rate, relatively poor efficacy precludes use of ampicillin for empirical treatment of uncomplicated UTI.

The only encouraging result obtained in our study was that the level of resistance of $E$. coli to aminoglycosides was relatively low (gentamicin 20.14\% and amikacin 5.41\%), as well as to the third generation cephalosporins (a little over 7\%), which, according to the IDSA recommendations (9), should be used for severe cases of UTI. Some of these drugs are not available in the oral form, so parenteral administration bears inconvenience, and is also more expensive option for the UTI treatment.

The characteristics of patients with UTI, such as age, are of great importance when deciding about the most suitable empirical treatment for uncomplicated UTI. Previous study have shown the impact of age on resistance of isolates from urine (22). Also, total microbe resistance of most common pathogens isolated from urine samples in women with symptoms of UTI, particularly of E. coli, was shown as significantly higher for mainly used antimicrobial drugs for community-acquired UTIs (e.g., ampicillin, trimethoprim-sulphamethoxazole, amoxicillin/clavulanic acid, nitrofurantoin, cefuroxime, ciprofloxacin) in the postmenopausal (older) women compared to premenopausal women (23). In a study conducted by Gobernado et al. (24), higher resistance rates of E. coli in older women (aged $>65$ years) were also found: resistance to ampicillin was $56 \%$ in the subgroup of women $>65$ years old, in comparison to $50 \%$ for women below that age $(\mathrm{OR}=1.2$; $95 \% \mathrm{CI}=2.2-3.5 ; \mathrm{p}=0.02)$; for ciprofloxacin the rates were $29 \%$ vs. $13 \%$, respectively $(\mathrm{OR}=2.8 ; 95 \% \mathrm{CI}=1.0-1.5 ; \mathrm{p}<0.001)$, and for co-trimoxazole $32 \%$ vs. $23 \%$, respectively ( $\mathrm{OR}=1.6 ; 95 \%$ $\mathrm{CI}=1.3-2.0 ; \mathrm{p}<0.001)$.

In our study, resistance rates of $E$. coli were statistically significantly higher in the group of the oldest women ( $>65$ years of age) in comparison to the two younger study groups, and it was observed for all tested antibiotics (cephalexin, TMP-SXT as well as ciprofloxacin). The prevalence of isolates resistant to all three observed antibiotics was also higher among isolates from elderly females (aged $>65$ years). Due to higher incidence of UTIs in older patients (25) and increased empirical use of antibiotics, higher rates of antibiotic resistance can be considered as one of the features of older adults.

The obtained results suggest that empirical therapy for uncomplicated UTI in our region should be individually adjusted, so TMP-SXT should not be used in women older than 65 years, and in younger ones only if the sensitivity of the isolate is known. TMP-SMX should not be used empirically if the patient has received this agent for treatment of UTI during the previous 3 months. Due to the high resistance to TMP-SXT fluoroquinolones actually become the first-line treatment of uncomplicated UTIs in our region, especially for older women, which is contrary to recommendations from the guidelines that they should be reserved for more invasive infections. Widespread use of fluoroquinolones for so common and mild infections could accelerate the development of resistance. Furthermore, although the fosfomycin is registered for oral use, it is unfrequently used in Serbia. It is also an appropriate choice for initial therapy, where available, because of minimal resistance and propensity for collateral damage. Such situation dictates the need for urgent adoption of the national guidelines for empirical therapy of UTIs.

Especially worrying result of this study is the fact that less than $50 \%$ of $E$. coli isolates were sensitive to all three tested antibiotics, and nearly $13 \%$ acquired triple-resistance. The prevalence of isolates resistant to two or three agents was higher in the subgroup of women older than 65 years. This could be explained partly by risk factors such as frequent use of antibiotics or presence of urinary catheter in the elderly (26). Furthermore, the observed unusual TMP-SXT-resistant antibiotype without resistance to other classes of antibiotics and increasing rates of TMP-SXT resistance become more common as the resistance rates to cephalexin or ciprofloxacin increase. It is known that resistance to TMP-SXT has been associated with concomitant resistance to other antibiotics.

From the graphical representation of the trend in resistance rate of $E$. coli to antibiotics we may see that, despite minor variations, it is growing, and that for ciprofloxacin almost doubled during the follow-up period (from $17.93 \%$ in 2009 to $34.46 \%$ in the last year of observation). As the increasing resistance of Enterobacteriaceae is often associated with higher use of antibiotics $(27,28)$, we tried to confirm this assumption in our study. After expressing utilization of antibiotics in DDD/1,000 inhabitants/ day, we tried to correlate it with the resistance rates. However, we did not find convincing evidence of correlation between the resistance rate of $E$. coli and utilization of antibiotics. This could be partly explained by the fact that the data on DDD were based on the whole community, not just on women. It may be assumed that the relationship between resistance and antibiotic utilization is probably influenced by additional factors and it cannot be explained only by utilization rate.

The results presented in our paper are in line with those presented in several recent papers. Thus, Bergman et al. (29) in a study conducted during a period of 9 years (from 1997 through 2005) and 20 hospital districts in Finland did not find significant association between fluoroquinolone use and resistance of E. coli to fluoroquinolones. On the contrary, previous study (30) found that development of $E$. coli resistance to fluoroquinolones correlated significantly with their utilization in the community $(r=0.859, p=0.029)$, which supports the need of controlled use of these effective antibiotics.

Often irrational and improper use of antibiotics in human and veterinary medicine leads to an alarming increase in the microbial resistance to antibiotics, which has become a serious public health problem. If we know that approximately $15 \%$ of all communityprescribed antibiotics are dispensed for UTI and that in the next 10 years only a few new antibiotics will be developed, prudent use of available antibiotics is the only option to delay the development of resistance (31) and the urological community has responsibility to participate in these efforts. Therefore, it is necessary to follow the IDSA guidelines when treating UTIs. 


\section{CONCLUSIONS}

Empirical choice of antimicrobial agent for communityacquired non-complicated UTIs in women should be individualized on the basis of the patient's age, prevalence of resistance in the local community, and compliance history of the patient. In addition, our data could also help to health authorities with formulation of national recommendations for treatment of UTIs and rational use of antibiotics. TMP-SXT should not be used for UTIs in elderly women, and ampicillin in all age groups. Empirical use of fluoroquinolone and cephalosporines of the first and second generation should be limited in the outpatient settings, in order to reconstitute susceptibility of $E$. coli to these important groups of antibiotics.

\section{Acknowledgements}

This study was partially financed by Research grant No. 175007 awarded by the Serbian Ministry of Education, Science and Technological Development.

\section{Conflict of Interests}

None declared

\section{REFERENCES}

1. Schappert SM, Rechtsteiner EA. Ambulatory medical care utilization estimates for 2007. Vital Health Stat 13. 2011;(169):1-38.

2. Salvatore S, Salvatore S, Cattoni E, Siesto G, Serati M, Sorice P, et al. Urinary tract infections in women. Eur J Obstet Gynecol Reprod Biol. 2011;156(2):131-6.

3. Naber KG, Schito G, Botto H, Palou J, Mazzei T. Surveillance study in Europe and Brazil on clinical aspects and Antimicrobial Resistance Epidemiology in Females with Cystitis (ARESC): implications for empiric therapy. Eur Urol. 2008;54(5):1164-75.

4. Hooton TM. Uncomplicated urinary tract infection. N Engl J Med. 2012;366(11):1028-37.

5. Guay DR. Contemporary management of uncomplicated urinary tract infections. Drugs. 2008;68(9):1169-205.

6. Wagenlehner FM, Weidner W, Naber KG. An update on uncomplicated urinary tract infections in women. Curr Opin Urol. 2009;19(4):368-74.

7. Clinical and Laboratory Standard Institute. Performance standards for antimicrobial susceptibility testing. Wayne: CLSI; 2010.

8. Agency for Medicines and Medical Devices of Serbia [Internet]. [cited 2016 Apr 10]. Available from: http://www.alims.gov.rs/latin/o-agenciji/ publikacije/. (In Serbian.)

9. Gupta K, Hooton TM, Naber KG, Wullt B, Colgan R, Miller LG, et al. International clinical practice guidelines for the treatment of acute uncomplicated cystitis and pyelonephritis in women: a 2010 update by the Infectious Diseases Society of America and the European Society for Microbiology and Infectious Diseases. Clin Infect Dis. 2011;52(5):e10320. doi: $10.1093 / \mathrm{cid} / \mathrm{ciq} 257$.

10. Grabe M, Bjerklund-Johansen TE, Botto H, Çek M, Naber KG, Pickard RS, et al. Guidelines on Urological Infections. European Association of Urology; 2013.

11. Farrel DJ, Morrisey I, De Rubeis D, Robbins M, Felmingham D. A UK multi-center study of the antimicrobial susceptibility of bacterial pathogens causing urinary tract infection. J Infect. 2003;46(2):94-100.

12. Kahlmeter G, Poulsen HO. Antimicrobial susceptibility of Escherichia coli from community-acquired urinary tract infections in Europe: the ECO-SENS study revisited. Int J Antimicrob Agents. 2012;39(1):45-51.

13. Gyssens IC. Antibiotic policy. Int J Antimicrob Agents. 2011;38 Sup$\mathrm{pl}: 11-20$.
14. Kahlmeter G, Åhman J, Matuschek E. Antimicrobial resistance of Escherichia coli causing uncomplicated urinary tract infections: a European update for 2014 and comparison with 2000 and 2008. Infect Dis Ther. 2015;4(4):417-23.

15. Schito GC, Naber KG, Botto H, Palou J, Mazzei T, Gualco L, et al. The ARESC study: an international survey on the antimicrobial resistance of pathogens involved in uncomplicated urinary tract infections. Int $\mathrm{J}$ Antimicrob Agents. 2009;34(5):407-41.

16. Meumann EM, Mitchell BG, McGregor A, McBryde E, Cooley L. Urinary Escherichia coli antimicrobial susceptibility profiles and their relationship with community antibiotic use in Tasmania, Australia. Int J Antimicrob Agents. 2015;46(4):389-93.

17. McIsaac WJ, Moineddin R, Meaney C, Mazzulli. Antibiotic-resistant Escherichia coli in women with acute cystitis in Canada. Can J Infect Dis Med Microbiol. 2013;24(3):143-9.

18. Meier S, Weber R, Zbinden R, Ruef C, Hasse B. Extended-spectrum $\beta$-lactamase-producing Gram-negative pathogens in community-acquired urinary tract infections: an increasing challenge for antimicrobial therapy. Infection. 2011;39(4):333-40.

19. Nicolle LE. Update in adult urinary tract infection. Curr Infect Dis Rep. 2011;13(6):552-60.

20. Wagenlehner FM, Schmiemann G, Hoyme U, Fünfstück R, HummersPradier E, Kaase M, et al. National S3 guideline on uncomplicated urinary tract infection: recommendations for treatment and management of uncomplicated community-acquired bacterial urinary tract infections in adult patients.Urologe A. 2011;50(2):153-69.

21. Gupta K, Sahm DF, Mayfield D, Stamm WE. Antimicrobial resistance among uropathogens that cause community-acquired urinary tract infections in women: a nationwide analysis. Clin Infect Dis. 2001;33(1):89-94.

22. Alós JI, Serrano MG, Gómez-Garcés JL, Perianes J. Antibiotic resistance of Escherichia coli from community-acquired urinary tract infections in relation to demographic and clinical data. Clin Microbiol Infect. 2005;11(3):199-203.

23. Miotla P, Romanek-Piva K, Bogusiewicz M, Markut-Miotla E, Adamiak A, Wróbel A, et al. Antimicrobial resistance patterns in women with positive urine culture: does menopausal status make a significant difference? Biomed Res Int. 2017;2017:4192908. doi: 10.1155/2017/4192908.

24. Gobernado M, Valdés L, Alós JI, García-Rey C, Dal-Ré R, García-deLomas J; Spanish Surveillance Group for Urinary Pathogens. Antimicrobial susceptibility of clinical Escherichia coli isolates from uncomplicated cystitis in women over a 1-year period in Spain. Rev Esp Quimioter. 2007;20(1):68-76

25. Rowe T, Juthani-Mehta M. Urinary tract infection in older adults. Aging Health. 2013; 9(5). doi: 10.2217/ahe.13.38.

26. Matthews SJ, Lancaster JW. Urinary tract infections in the elderly population. Am J Geriatr Pharmacother. 2011;9(5):286-309.

27. Sedláková MH, Urbánek K, Vojtová V, Suchánková H, Imwensi P, Kolár $\mathrm{M}$. Antibiotic consumption and its influence on the resistance in Enterobacteriaceae. BMC Res Notes. 2014;7:454. doi: 10.1186/17560500-7-454.

28. Joseph NM, Bhanupriya B, Shewade DG, Harish BN. Relationship between antimicrobial consumption and the incidence of antimicrobial resistance in Escherichia coli and Klebsiella pneumoniae isolates. J Clin Diagn Res. 2015;9(2):DC08-12.

29. Bergman M, Nyberg ST, Huovinen P, Paakkari P, Hakanen AJ; Finnish Study Group for Antimicrobial Resistance. Association between antimicrobial consumption and resistance in Escherichia coli. Antimicrob Agents Chemother. 2009;53(3):912-7.

30. Urbánek K, Kolár M, Strojil J, Koukalová D, Cekanová L, Hejnar P. Utilization of fluoroquinolones and Escherichia coli resistance in urinary tract infection: inpatients and outpatients. Pharmacoepidemiol Drug Saf. 2005;14(10):741-5.

31. Guneysel O, Onur O, Erdede M, Denizbasi A. Trimethoprim/sulfamethoxazole resistance in urinary tract infections. J Emergency Med. 2009;36(4):338-41. 